\title{
An approach of adaptive notch filtering design for electrocardiogram noise cancellation
}

\author{
Rahmad Hidayat ${ }^{1}$, Ninik Sri Lestari ${ }^{2}$, Herawati $^{3}$, Givy Devira Ramady ${ }^{4}$, Sudarmanto ${ }^{5}$, Farhan Adani $^{6}$ \\ ${ }^{1,4}$ Department of Electrical Engineering, Sekolah Tinggi Teknologi Mandala, Indonesia \\ ${ }^{2}$ Department of Informatics Engineering, Sekolah Tinggi Teknologi Mandala, Indonesia \\ ${ }^{3}$ Department of Electrical Engineering, Universitas Kristen Maranatha, Indonesia \\ ${ }^{5}$ PT Astindo Dot Net, Harco Mangga Dua, Jakarta, Indonesia \\ ${ }^{6}$ Department of Informatics Engineering, Institut Teknologi Nasional, Indonesia
}

\section{Article Info \\ Keywords: \\ ECG \\ MATLAB \\ NLMS \\ Noise \\ Notch}

Article history:

Received Dec 10, 2020

Revised Apr 4, 2021

Accepted Apr 27, 2021

\begin{abstract}
An electrocardiogram (ECG) is a means of measuring and monitoring important signals from heart activity. One of the major biomedical signal issues such as ECG is the issue of separating the desired signal from noise or interference. Different kinds of digital filters are used to distinguish the signal components from the unwanted frequency range to the ECG signal. To address the question of noise to the ECG signal, in this paper the digital notch filter IIR $47 \mathrm{~Hz}$ is designed and simulated to demonstrate the elimination of $47 \mathrm{~Hz}$ noise to obtain an accurate ECG signal. The full architecture of the structure and coefficient of the IIR notch filter was carried out using the FDA Tool. Then the model is finished with the help of Simulink and the MATLAB script was to filter out the $47 \mathrm{~Hz}$ noise from the signal of ECG. For this purpose, the normalized least mean square (NLMS) algorithm was used. The results indicate that before being filtered and after being filtered it clearly shows the elimination of $47 \mathrm{~Hz}$ noise in the signal of the ECG. These results also show the accuracy of the design technique and provide an easy model to filter out noise in the ECG signal.
\end{abstract}

This is an open access article under the CC BY-SA license.

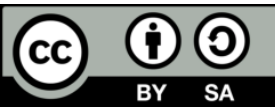

Corresponding Author:

Rahmad Hidayat

Department of Electrical Engineering

Sekolah Tinggi Teknologi Mandala

597 Soekarno Hatta St., Bandung 40286, Indonesia

Email: rhidayat4000@gmail.com

\section{INTRODUCTION}

The signals are interpreted in digital signal processing by mathematical functions such as sinusoidal functions or linear equations of differences. Electrocardiogram signal widely used has been to detect the human heartbeat and is useful for heart pathologies. One of the major biomedical signal issues such as an electromyogram (EMG) and an electrocardiogram (ECG) is denoising issues. Especially for ECG, the issue is the distinction between the desired signal and the noise or interference caused by the 49 to $51 \mathrm{~Hz}$ powerline interference, muscle artifacts, wandering, or electrode artifacts [1], [2]. Besides that, for an ECG signal of $50 \mathrm{~Hz}$, frequencies from $47 \mathrm{~Hz}$ to $53 \mathrm{~Hz}$ are more important. This may occur due to fluctuations in the hospital power supply from $47 \mathrm{~Hz}$ to $53 \mathrm{~Hz}$. [3] For the ECG signal, these frequencies can be noise and the efficiency of ECG recording can be diminished because of it. Therefore nothing appears to be safer than using adaptive filters to resolve the lack of information in the ECG recording [4]. Meanwhile, various types of adaptive digital filters have been used to separate the unwanted-frequency signal element ranges and matched them for random processes [2], [5]. 
MATLAB is the most useful software kit for engineering and application implementation environments. It is used for particular purposes in various fields of electronic programming related technology, a graphical illustration of science \& engineering, precise numerical measurement, and development of algorithms [6]-[9]. This research purposes to design a notch filter to be able to eliminate 47 $\mathrm{Hz}$ noise interference sources with the adaptive filter algorithm of normalized least mean square (NLMS) on the ECG system and simulate it using Simulink available in MATLAB.

Notch filter must satisfy many criteria for a successful digital signal processor (DSP) scheme, i.e. it must respond to all input frequencies, it should be robust, more efficient for signal manipulation. The most significant aim for adaptive filter selection was its ability to modify the filter coefficients, and how to identify rules or algorithms that upgrade coefficients was the key designating element. The adaptive filters measure signal efficiency and also monitor the signal, improve the solution, and decide how to update the coefficients of filters. It suppresses signals in a certain frequency range within the bandstop filter (band-reject filter). It has two bands and a stopband in it. If the stopband is very narrow, it is called a notch filter. The transfer function of the notch is shown in (1).

$$
H(z)=\left(1-a z^{-1}+z^{-2}\right) /\left(1-a r z^{-1}+r^{2} z^{-2}\right)
$$

Where $\mathrm{a}=2 \cos \omega$, and $\mathrm{r}<|1|$. The parameter $\mathrm{r}$ determines the pole distance from the unit circle and the normalized bandwidth, where $\mathrm{r}$ is reversely proportional to the reciprocal bandwidth of the notch filter. In addition to the ECG system, notch filters are used in various fields including estimation systems [10], [11].

Adaptive noise cancellation (ANC) is an alternate calculation methodology for noise or interference in a corrupted signal. The main purpose of noise cancellation is to assess the noise and reduce it from the combination of the original input signal and the noise signal so that a noise-free signal is obtained. In adaptive noise cancellation, additional noise is given to measure the damaged input signal. The reference input is adaptive filtering and subtraction from the main input signal to obtain an estimated signal. In this method, the desired signal (which is damaged by an additional noise) can be recovered using a certain algorithm. [12], [13] Various kinds of adaptive algorithms are used for noise canceling like least mean square (LMS), normalized LMS (NLMS), recursive least square (RLS), including interference cancellation algorithm of multi-stage partial parallel [14], [15]. The LMS algorithm provides good numerical stability and has few hardware requirements, however, the weakness of this algorithm is in terms of convergence. Meanwhile, one of the NLMS algorithms is the most widely used algorithms in technical fields. An updated version of the regular LMS algorithm is the NLMS algorithm. Using the following by way of the theorem, updating the coefficients of the adaptive filter with the NLMS algorithm [12], [16]:

$$
\bar{w}(n+1)=\bar{w}(n)+\mu e(n) \frac{\bar{u}(n)}{\|\bar{u}(n)\|^{2}}
$$

That may have been written as,

$$
\bar{w}(n+1)=\bar{w}(n)+\mu(n) e(n) \bar{u}(n)
$$

where

$$
\mu(n)=\mu\|\bar{u}(n)\|^{2}
$$

In the earlier equivalence, the algorithm of NLMS is similar to the regular LMS algorithm, except that there is a time-varying NLMS algorithm step size of $\mu(n)$. The step size $\mu(n)$ determines the speed and stability of the adaptation. The adaptive filter's convergence speed can be enhanced by this phase scale. Compared to the algorithm for LMS, the algorithm of NLMS is a theoretically faster converging algorithm, which can come at a higher residual error price. The key downside of the pure LMS algorithm is that it is vulnerable to its Input xx scaling (n). This makes it very difficult to select a $\mu$ learning rate that maintains algorithm consistency. The NLMS is a modified of the LMS algorithm that fixes this tricky by normalizing the input power $[12,17]$. Flowchart of adaptive filtering algorithm as shown in Figure 1.

Heart rate frequency is very relevant data for health status. In certain medical or sports uses, such as stress checks or life care condition estimation, the frequency calculation is used. Calculating it from the signal of ECG is one of the potential methods of accessing heart rate frequency. The electrocardiogram (ECG) represents the human heart's electrical function. The ECG consists of five waves - P, Q, R, S, and T. This signal could be assessed by the usual presence of electrodes in the human body. With amplifiers and analog-digital converters, signals from these electrodes are carried into basic electrical circuits. Many 
methods and algorithms can detect the heart rate frequency from the ECG signal. Many heart rate detection algorithms are based on the detection of QRS complexes, and heart rate is measured as the interval between complexes of QRS. For example, the complexity of QRS can be defined using artificial neural network algorithms, genetic algorithms, wavelet transforms, or filterbanks [18] The results of the ECG screening provide the possibility of analyzing heart disease. ECG signal as shown in Figure 2.

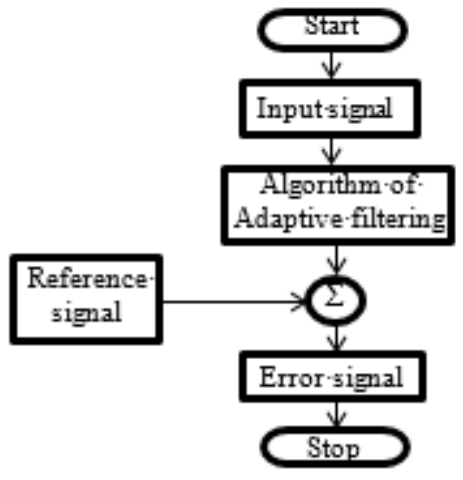

Figure 1. Flowchart of adaptive filtering algorithm

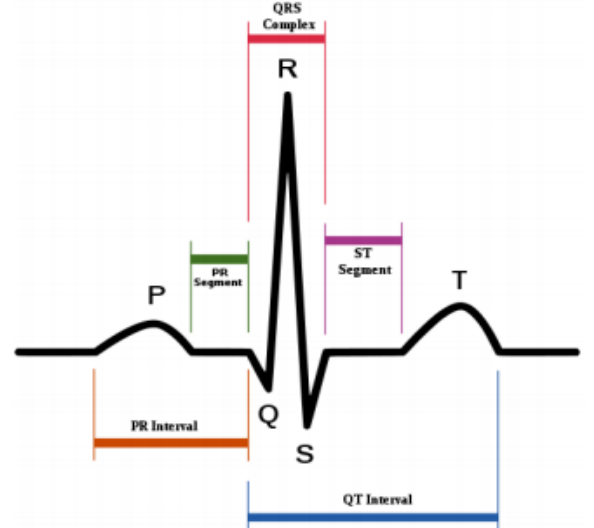

Figure 2. ECG signal [2]

In the work of [19], a digital FIR filter is developed and applied with various windowing techniques such as Hamming, Kaiser, and Chebyshev to eliminate $50 \mathrm{~Hz}$ power line noise in the signal of ECG. The assessment in the MATLAB setting was completed. Comparing the waveforms of the initial and filtered ECG signals, for all FIR filters, the results obtained are compared with. The one who uses the Chebyshev window is the filter that provides the best results. Detection, estimation, and filtering of the desired signal against noise are some of the most common methods. The time-domain analysis also requires a comparison of two distinct signals in which a stochastic signal is usually more advantageously analyzed in the time domain. Through certain implementations of signal processing, there is the breakdown of the original signal into the fundamental signal. The notch adaptive filter can extract the noise from the target signal. Another way to reduce noise can also be done with another filter called the Kalman filter [20]. Mane and Agashe [21], the proposed notch adaptive filter is connected in parallel to extract the major frequency of the noisecontaminated signal. Each filter is capable of decomposing the ' $n$ ' sinusoids which are harmoniously related to their constituent components.

Verma and Singh [22] proposed a high-performance tuneable adaptive notch filter algorithm for estimating the accuracy of ECG signals. Using the proposed notch adaptive filter with FIR tunable notch frequencies, an interference of electrical wire and the noise of muscle contraction is greatly suppressed. The conservation of selectivity and attenuation at notch frequencies is an essential feature of the suggested filter scheme. The filters are optimized and their coefficients are calculated such that noise in the signal of ECG is minimized within the specified frequency range. The proposed algorithm estimates the frequency of the unwanted signal and updates the filter consistent with the filter coefficient for optimal performance. Designing an ECG machine using ATmega microcontroller technology has been carried out in a study [23] where the error rate is still below the maximum allowable threshold.

Computer simulations based on digital equivalents are a feasible and real answer for the acquisition and extraction of human bio-signals which are very sensitive to interference. But these digital filters are mostly verified on stored databases so there is a need to improve the acquired system in real-time. Research [24] deals with the design and simulation of IIR notch filters for implementation on real-time, noninvasive acquired carotid pulse waves with Simulink and the FDA Tool. To analyze an ECG signal with different frequency components, a wavelet-decomposition-filter reconstruction (WDFR) algorithm is employed in [25]. The algorithm was applied for eliminating noise and artifact components in ECG signals. The NLMS algorithm has been simulated in [20] that NLMS has advantages in terms of mean square error (MSE) compared to the LMS and RLS algorithms [26]. For simulation purposes, additive white Gaussian noise is added to the information signal generated randomly and efficiently reduces noise with minimum or no errors, and the NLMS algorithm is used to reach the desired result as is done in [27] and [12]. Also, the noise cancellation efficiency of the NLMS was consistently higher compared to the ECG signal ANC LMS algorithm. [3] NLMS was also used in research [28] as a continuation of the adaptive line enhancer (ALE) 
filters for the adaptive noise cancellation scheme. To avoid manual calculation of PQRST peak amplitude values, the software designed in [29] has been able to represent the PQRST and cardiogram peak amplitude values for each cycle on each electrocardiogram lead. The results of the continuous signal electrocardiogram (ECG) examination were sampled at a certain frequency to obtain discrete data which is the amplitude as a function of integer (N). Adaptive filter estimation of an ANC can be done using MATLAB and/or Simulink. For this reason, the MATLAB software was used in the study of [30] to efficiently detect any abnormalities existing in the ECG signal. The detection of such abnormalities refers to the $\mathrm{P}, \mathrm{Q}, \mathrm{R}$, and $\mathrm{S}$ peaks of the ECG signal.

\section{RESEARCH METHOD}

The simulation parameters of this study, both for the notch filter design and for the simulink block, are listed in Table 1. The algorithm that we use is shown in Figure 3. While the notch simulation configuration are shown in Figure 4. The simulation is also complemented by using the MATLAB script with 32 filter taps.

Table 1. The simulation parameters

\begin{tabular}{llll}
\hline \multicolumn{2}{c}{ Filter Design } & (FDA Tool) & Simulink \\
\hline Filter designed: & Notch & Sine wave: & \\
Frequency: & $47 \mathrm{~Hz}$ & amplitude: & $1 \mathrm{volt}$ \\
Type: & IIR & frequency: & $50 \mathrm{~Hz}$ \\
Structure: & Direct form II & phase offset: & $0 \mathrm{rad}$ \\
Order: & Second order & sample mode: & discrete \\
$\mathrm{BW}_{3 \mathrm{~dB}}:$ & $80 \mathrm{~Hz}$ & sample per frame: & 1 \\
$\mathrm{~A}_{\text {pass: }}$ & $1 \mathrm{~dB}$ & Noise source: & \\
Sampling frequency: & $360 \mathrm{~Hz}$ & type: & Gaussian (Ziggurat) \\
& & mean: & 0 \\
& & variance: & 1 \\
& & the initial seed: & {$[23341]$} \\
& & sample mode: & discrete \\
& & SNLMS filter: & \\
& & algorithm: & Normalized LMS \\
& & filter length: & 32 \\
& Sample time: & $1 / 360 \mathrm{~s}$ \\
& Solver simulation: & fixed-step with discrete \\
& & & (no continuous states) \\
& & Duration: & 600 seconds \\
\hline
\end{tabular}

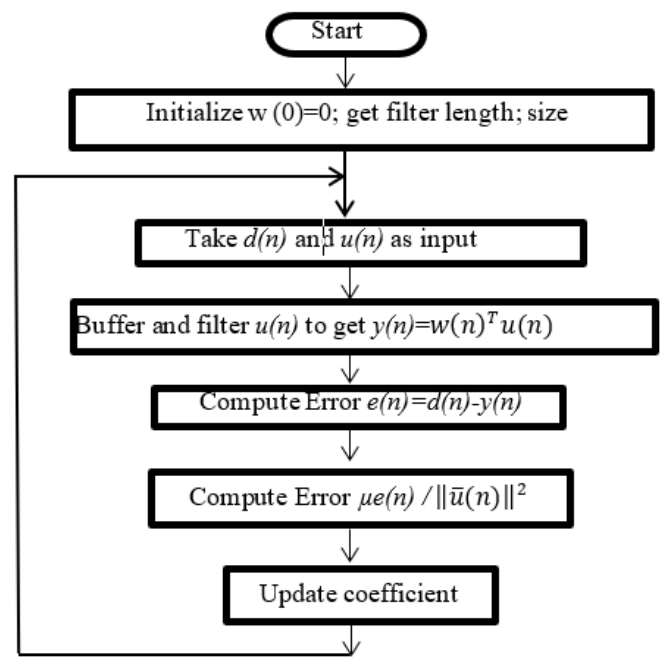

Figure 3. NLMS algorithm flowchart [3] 


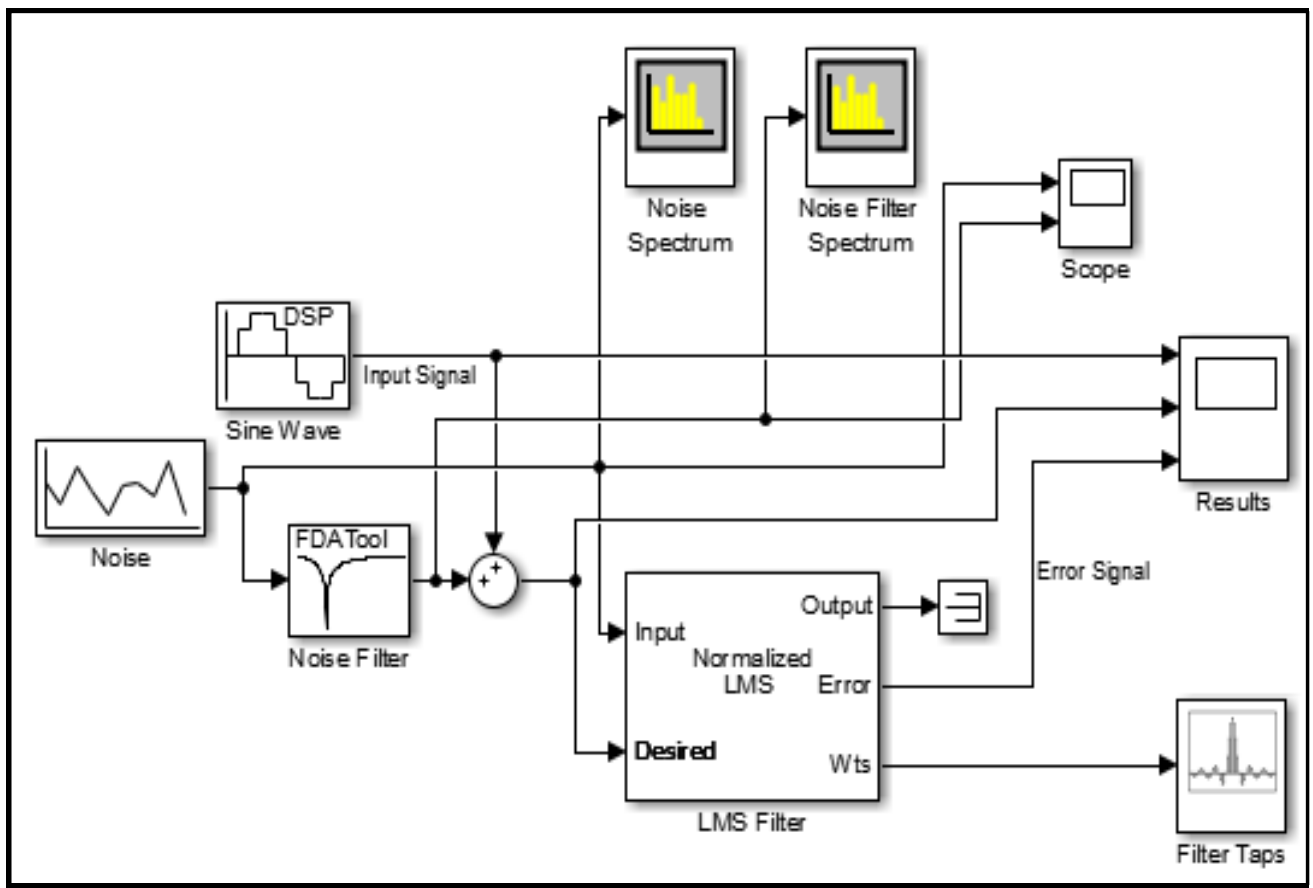

Figure 4. The simulink block

\section{RESULTS AND DISCUSSION}

\subsection{Filter design result}

According to filter analysis, there are several other parameters such as a pole, zero, a (the value of $2 \cos \mathrm{w}$ ), $\mathrm{r}$ (i.e. a constant slightly less than 1 , such that the poles are located in the realm of equilibrium), and the sampling frequency which affects the performance of the notch filter. The notch filter is displayed in Figure 5 designed for a bandwidth of $80 \mathrm{~Hz}$ to guarantee the Nyquist theorem at the sampling frequency and notch frequency taken in this study.

In measuring filter performance, filter stability is very important. The filter poles must remain in the loop to ensure the filter output stability. All the poles in this notch filter design are in the unit circle. This shows that the planned notch filter is stable. Also, the poles in Figure 6 relate to the resulting notch bandwidth. This is closely related to the narrow beam of Figure 5.

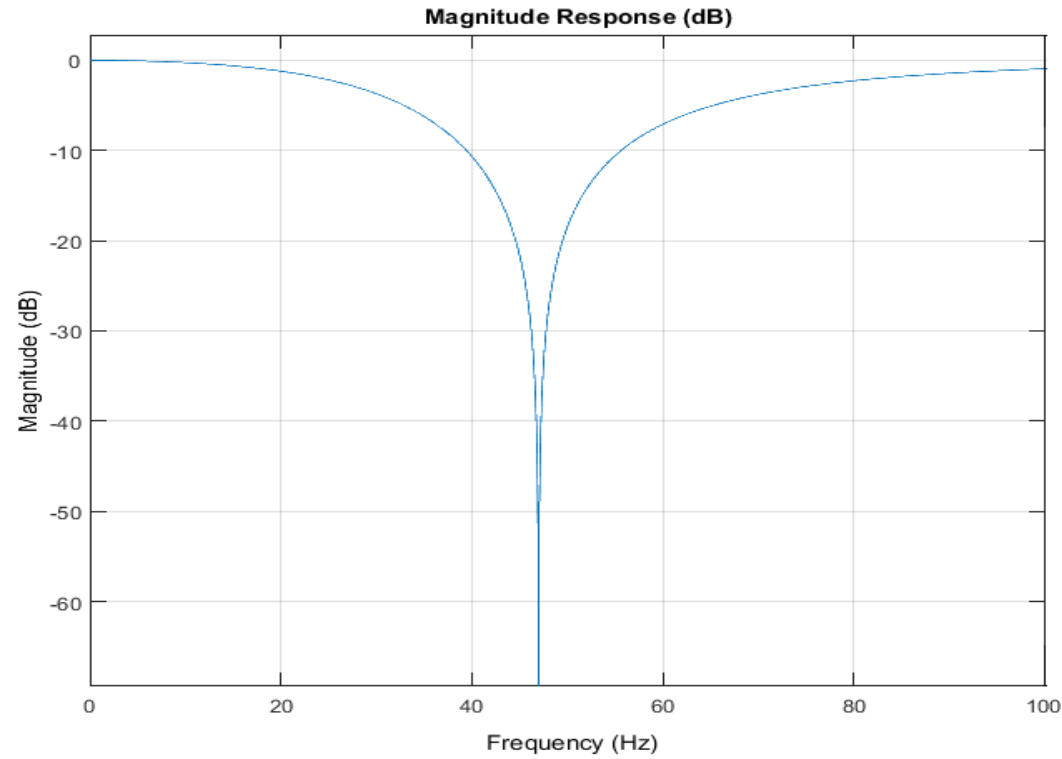

Figure 5. Notch designed $(47 \mathrm{~Hz})$ 


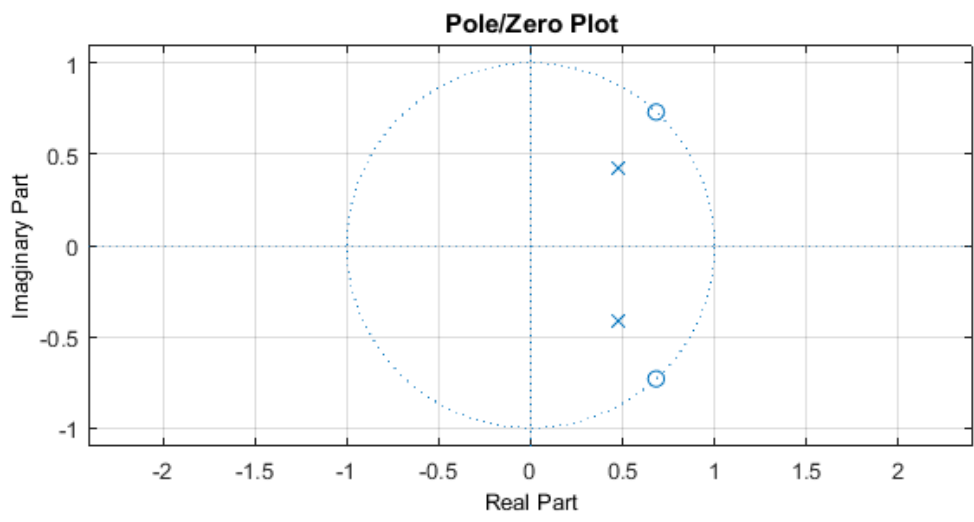

Figure 6. A plot of $47 \mathrm{~Hz}$ notch pole-zero

\subsection{Simulink result}

Figure 7 displays the output spectrum of the notch filter. The left-right cursors are the bandwidth range taken in this study, which is $80 \mathrm{~Hz}$. The level of the two points towards the lowest level of the spectrum shows that the $47 \mathrm{~Hz}$ notch filter can reduce the noise level average by $50.51 \mathrm{~dB}$. Filter taps as shown Figure 8. The taps filter is closely related to the notch filter impulse response which is designed where the magnitude decays towards zero. This ensures that the designed notch IIR filter is a stable system. The spectrum of 'result' as shown in Figure 9.

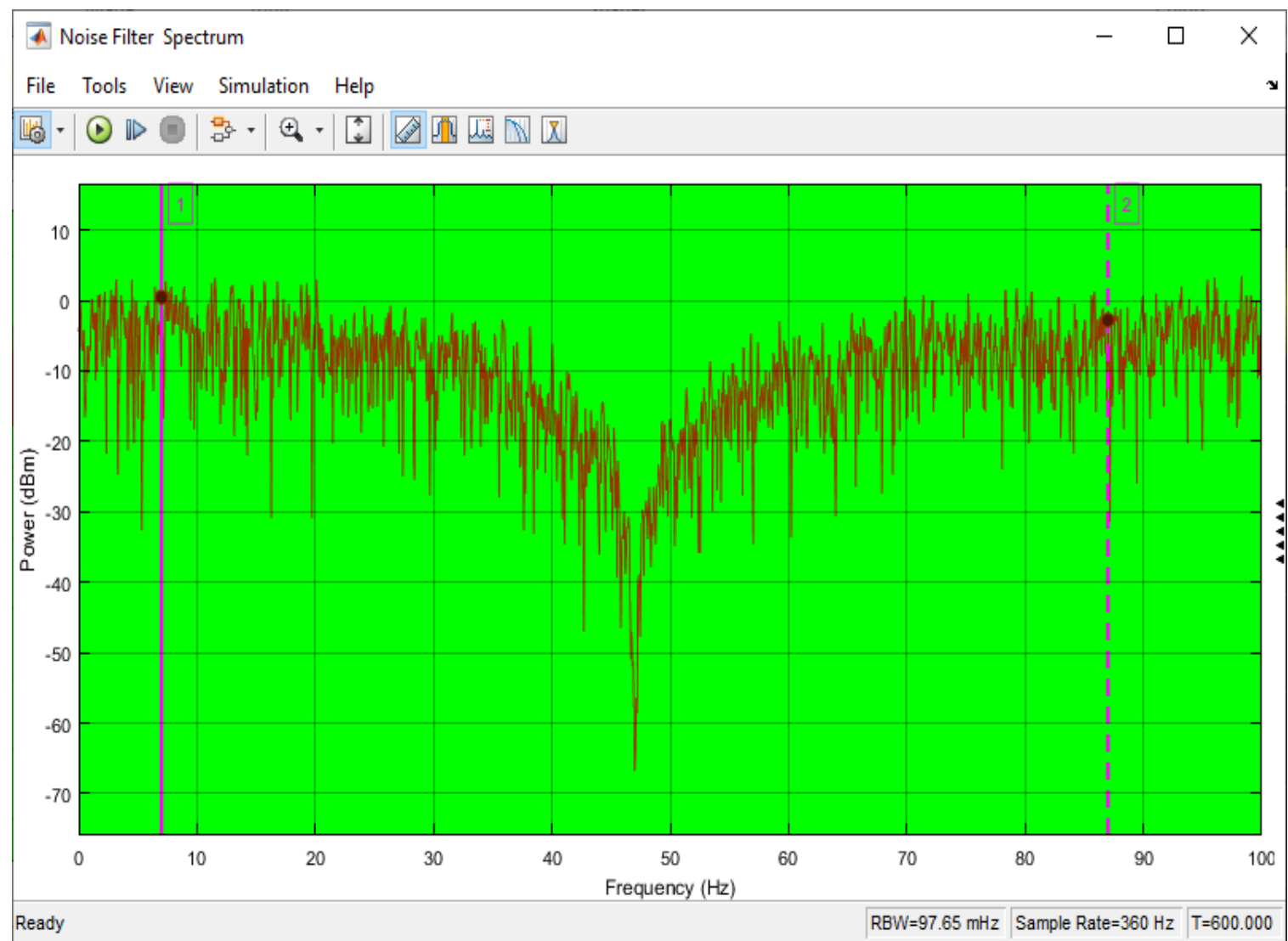

Figure 7. The spectrum of the noise filter 


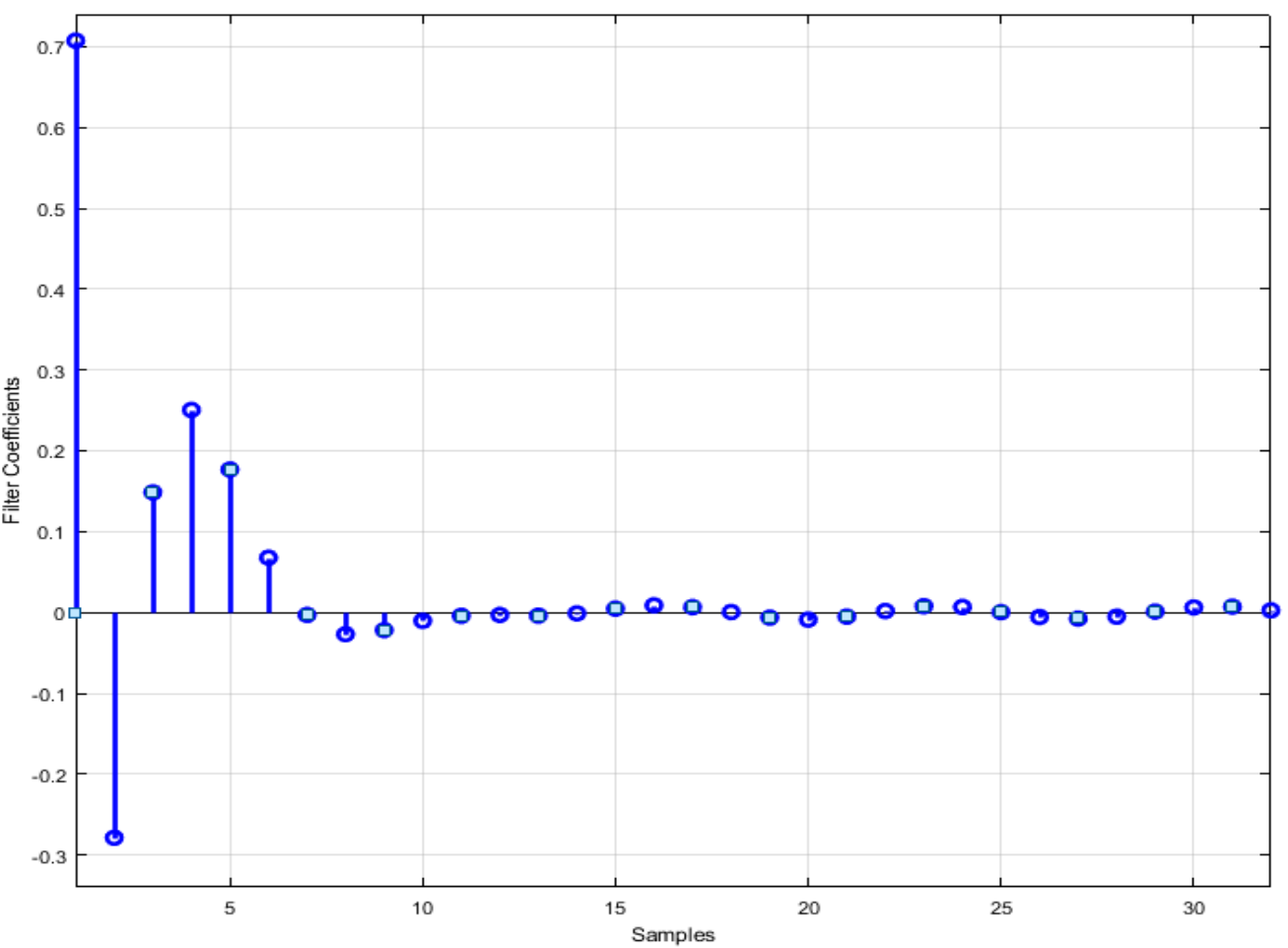

Figure 8. Filter taps
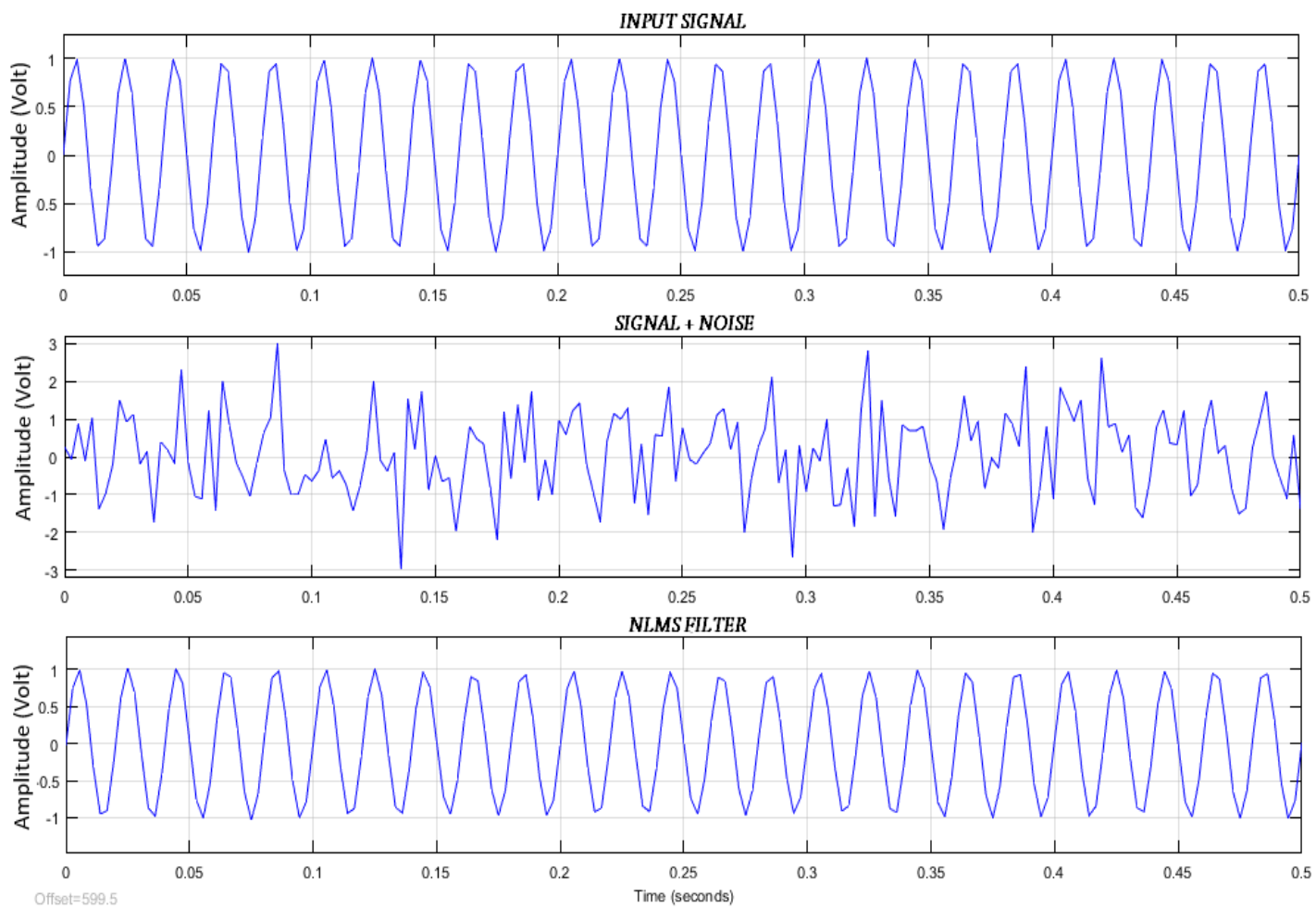

Figure 9. The spectrum of 'result'

The results of the "Error Signal" in the scope "Result" for some of the $\mu$ values get the optimum value of 0.01 . Like the simulink block in this study, one of the output ports of the NLMS filter is called "Error" and the signal is $e(n)$ which is called "Error Signal." This signal is an imitation of the $50 \mathrm{~Hz}$ input signal. With the NLMS algorithm used, it can be told that the imitation signal simulation is almost similar to 
the pure signal after the $47 \mathrm{~Hz}$ noise is removed. This indicates a successful adaptation process in the adaptive filtering system.

\subsection{MATLAB result}

The mixed-signal in Figure 10 is a combination of a clean ECG signal and $47 \mathrm{~Hz}$ noise. Meanwhile, the output signal from the filtering "Notch NLMS filter output" is shown in the fourth plot. It can be appreciated that the output shape of the filter with the algorithm of NLMS is like the original ECG signal. This means that the system adaptation process after removing $47 \mathrm{~Hz}$ noise from a $50 \mathrm{~Hz}$ ECG signal with this algorithm is almost perfect. This MATLAB simulation data includes Fs $=360 \mathrm{~Hz} ; \mu=0.01$; Filter tap $=16$.
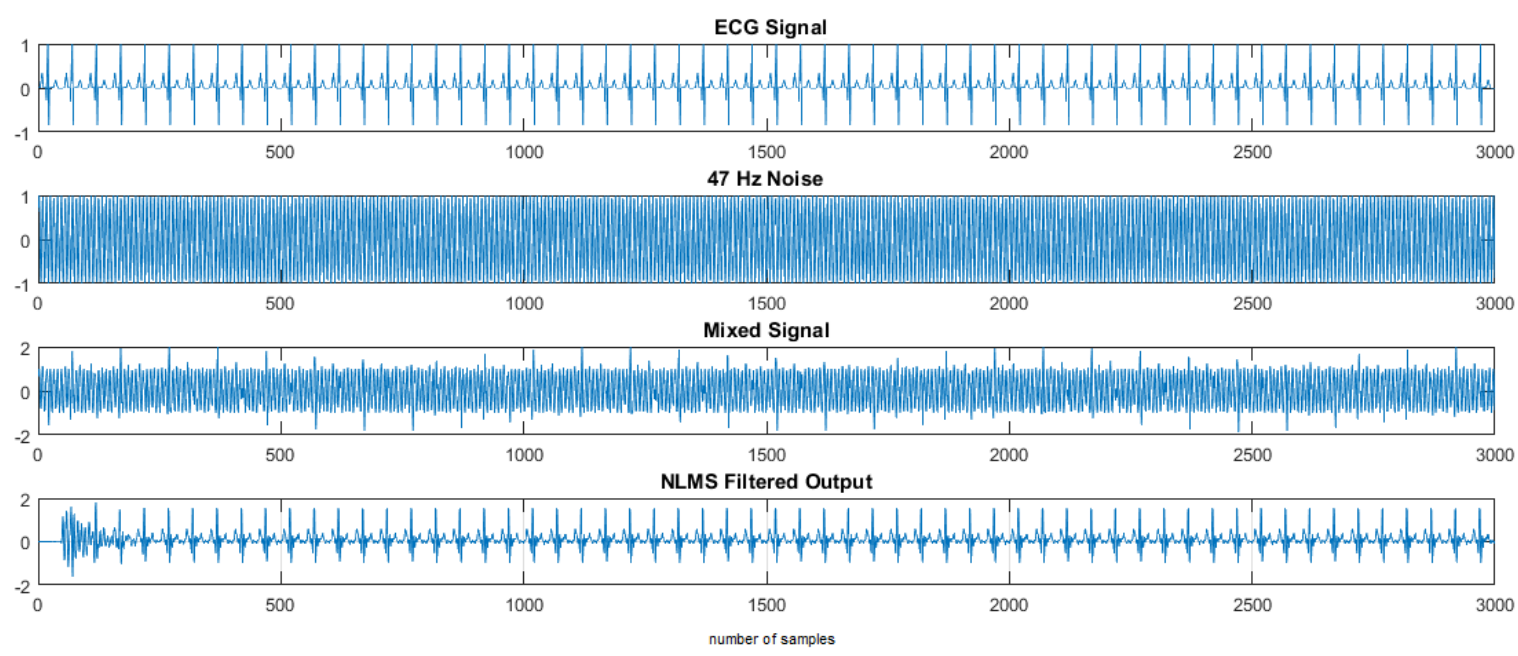

Figure 10. ECG noise filtering

\section{CONCLUSION}

Using the Simulink setting, the algorithm was developed and implemented using the normalized least mean square (NLMS) adaptive filter. To eliminate $47 \mathrm{~Hz}$ noise from the signal of ECG, notch adaptive filtering is chosen. The code is written for the input signal, which is expected to be a $50 \mathrm{~Hz}$ ECG signal and a second noise signal of $47 \mathrm{~Hz}$. To find the preferred performance, the effects of step size on convergence rate and stability for the NLMS adaptive filter were analyzed. The results showed that the filter designed was able to adapt to changes in frequency and response accordingly.

\section{REFERENCES}

[1] S. Elouaham, A. Dliou, M. Laaboubi, R. Latif, N. Elkamoun, and H. Zougagh, "Filtering and analyzing normal and abnormal electromyogram signals," Indones. J. Electr. Eng. Comput. Sci., vol. 20, no. 1, pp. 176-184, 2020, doi: 10.11591/ijeecs.v20.i1.pp176-184.

[2] M. Gachake, G. Gawande, and K. Khanchandani, "Performance Comparison of Various Digital Filters for Elimination of Power Line Interference from ECG Signal," Int. J. Curr. Eng. Technol., vol. 4, no. 3, pp. 1255-1259, 2014.

[3] H. K. Gupta, R. Vijay, and N. Gupta, "Designing and Implementation of Algorithms on MATLAB for Adaptive Noise Cancellation from ECG Signal," Int. J. Comput. Appl., vol. 71, no. 5, pp. 1-8, 2013, doi: 10.5120/123518652 .

[4] C. B. Mbachu and K. J. Offor, "Reduction of Powerline Noise in Ecg Signal Using Fir Digital Filter Implemented With Hamming Window," Int. J. Sci. Environ. Technol., vol. 2, no. 6, pp. 1380-1387, 2013.

[5] J. S. Sidhu and M. Singh, "Performance Comparison of Different Adaptive Filtering," IJCTA, vol. 9, no. 11, pp. 5281-5286, 2016.

[6] S. Purohit and M. K. Nagachandra, "Reconfigurable Antennas - Antennas that can," IJIRCCE, vol. 2, no. 4, pp. 4059-4064, 2014.

[7] M. R. Keshtkaran and Z. Yang, "A Fast, Robust Algorithm for Power Line Interference Cancellation in Neural Recording," J. Neural Eng. IOP, vol. 11, no. 2, 2014, doi: 10.1088/1741-2560/11/2/026017.

[8] P. Mendhe and N. Bodne, "Performance Evaluation of LMS, DLMS, and TVLMS Adaptive Filter," IJECET, vol. 7, no. 5, pp. 68-76, 2016.

[9] I. C. Deepika, "A Simulation \& Performance Analysis of ANC using Adaptive Filters," IJEIT, vol. 3, no. 2, pp. 283-287, 2013. 
[10] J. Levin, N. O. Pérez-arancibia, and P. A. Ioannou, "Adaptive Notch Filter Using Real-Time Parameter Estimation," IEEE Trans. Control Syst. Technol., vol. 19, no. 3, pp. 673-681, 2011, doi: 10.1109/TCST.2010.2049493.

[11] A. Elmelhi, "Modified Adaptive Notch Filter Based on Neural Network for Flexible Dynamic Control," Int. J. Comput. Electr. Eng., vol. 6, no. 2, pp. 185-190, 2014, doi: 10.7763/IJCEE.2014.V6.819.

[12] K. Sahu and R. Sinha, "Simulation of NLMS Adaptive Filter for Noise Cancellation," Int. J. Eng. Appl. Sci., vol. 2, no. 1, pp. 27-29, 2015.

[13] F. R. Jimenez-Lopez and C. E. Pardo-beainy, "Adaptive filtering implemented over TMS320c6713 DSP platform for system identification," ITECKNE, vol. 11, no. 2, pp. 157-171, 2014, doi: 10.15332/iteckne.v11i2.726.

[14] M. A. Patel and A. Rathod, "Adaptive Noise Cancellation," Golden Res. Thoughts, vol. 3, no. 10, 2014.

[15] Y. Liang, H. Wu, and G. Wang, "Multi-stage partial parallel interference cancellation algorithm for MUSA systems," TELKOMNIKA (Telecommunication Comput. Electron. Control), vol. 14, no. 4, p. 1390, 2016, doi: 10.12928/telkomnika.v14i4.4052.

[16] S. Singh, "A LMS and NLMS Algorithm Analysis for Smart Antenna," IJARCSSE, vol. 5, no. 4, 2015.

[17] F. Afroz, A. Huq, F. Ahmed, and K. Sandrasegaran, "Performance Analysis of Adaptive Noise Canceller Employing NLMS Algorithm," Int. J. Wirel. Mob. Networks, vol. 7, no. 2, pp. 45-58, 2015, doi: 10.5121/ijwmn.2015.7204.

[18] A. Bennet, Bhavani, and H. Priya, "Design and Analysis of Electrocardiograph ( ECG ) Signal for long term continuous heart rate monitoring system," Int. J. Pure Appl. Math., vol. 119, no. 15, pp. 99-110, 2018.

[19] A. Mittal and A. Rege, "Design of digital FIR filter implemented with window techniques for reduction of power line interference from ECG signal," in IC4, 2015, pp. 1-4, doi: 10.1109/IC4.2015.7375535.

[20] G. Badhwar, "Noise Reduction using Kalman Filter," IJLTET, vol. 7, no. 1, pp. 639-643, 2016.

[21] V. Mane and A. Agashe, "An Adaptive Notch Filter For Noise Reduction and Signal Decomposition," Int. J. Comput. Sci. Issues, vol. 8, no. 5, pp. 360-365, 2011.

[22] A. R. Verma and Y. Singh, "Adaptive Tunable Notch Filter for ECG Signal Enhancement," Procedia Comput. Sci., vol. 57, pp. 332-337, 2015, doi: 10.1016/j.procs.2015.07.347.

[23] B. G. Irianto, B. Budhiaji, and S. Syaifudin, "Design of electrocardiograph machine based on ATmega microcontroller," Indones. J. Electr. Eng. Comput. Sci., vol. 2, no. 2, pp. 328-333, 2016, doi: 10.11591/ijeecs.v2.i2.pp328-333.

[24] D. Bansal, "Computer Based Model to Filter Real Time Acquired Human Carotid Pulse," Signal Process. An Int. J., vol. 7 , no. 1, pp. 42-51, 2013.

[25] T. N. Nguyen, T. H. Nguyen, and V. T. Ngo, "Artifact elimination in ECG signal using wavelet transform," TELKOMNIKA (Telecommunication Comput. Electron. Control), vol. 18, no. 2, pp. 936-944, 2020, doi: 10.12928/telkomnika.v18i2.14403.

[26] G. K. Girisha and S. L. Pinjare, "Performance Analysis of Adaptive Filters for Noise Cancellation in Audio Signal for Hearing Aid Application," Int. J. Sci. Res., vol. 5, no. 5, pp. 364-368, 2016.

[27] M. K. Islam, A. N. M. M. Haque, G. Tangim, T. Ahammad, and M. R. H. Khondokar, "Study and Analysis of ECG Signal Using MATLAB \&LABVIEW as Effective Tools," Int. J. Comput. Electr. Eng., vol. 4, no. 3, pp. 404-408, 2012, doi: 10.7763/IJCEE.2012.V4.522.

[28] J. R. Mohammed and M. S. Shafi, "An Efficient Adaptive Noise Cancellation Scheme Using ALE and NLMS Filters," Int. J. Electr. Comput. Eng., vol. 2, no. 3, pp. 325-332, 2012, doi: 10.11591/ijece.v2i3.246.

[29] S. Setiawidayat and R. Joegijantoro, "Algorithm for the representation of parameter values of electrocardiogram," TELKOMNIKA (Telecommunication Comput. Electron. Control), vol. 16, no. 3, pp. 1295-1302, 2018, doi: 10.12928/telkomnika.v16i1.6934.

[30] D. K. Ojha and M. Subashini, "Analysis of Electrocardiograph ( ECG ) Signal for the Detection of Abnormalities Using MATLAB," Int. J. Medical, Heal. Biomed. Pharm. Eng., vol. 8, no. 2, pp. 114-117, 2014. 